\title{
Holocene sea-surface temperature variability in the Chilean fjord region
}

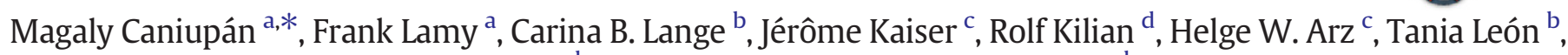 \\ Gesine Mollenhauer ${ }^{\mathrm{a}}$, Susana Sandoval ${ }^{\mathrm{b}}$, Ricardo De Pol-Holz ${ }^{\mathrm{e}}$, Silvio Pantoja ${ }^{\mathrm{b}}$, \\ Julia Wellner ${ }^{\mathrm{f}}$, Ralf Tiedemann ${ }^{\mathrm{a}}$ \\ a Alfred Wegener Institute for Polar and Marine Research, Am Alten Hafen 26, D-27568 Bremerhaven, Germany \\ ${ }^{\mathrm{b}}$ Department of Oceanography, Center for Oceanographic Research in the eastern South Pacific (COPAS), COPAS Sur-Austral Program, University of Concepción, Concepción, Chile \\ c Leibniz Institute for Baltic Sea Research Warnemünde, Seestraße 15, 18199 Rostock, Warnemünde, Germany \\ ' Department of Geology, FBVI, University of Trier, Behringstraße, D-54296 Trier, Germany \\ e Department of Oceanography, Center for Climate and Resilience Research (CR) ${ }^{2}$, University of Concepción, Concepción, Chile \\ ${ }^{\mathrm{f}}$ Department of Earth and Atmospheric Sciences, University of Houston, Houston, TX 77204, USA
}

\section{A R T I C L E I N F O}

\section{Article history:}

Received 6 December 2013

Available online 22 August 2014

\section{Keywords:}

Holocene

Alkenones

Sea-surface temperature

Fjords

Chile

\begin{abstract}
A B S T R A C T
Here we provide three new Holocene (11-0 cal ka BP) alkenone-derived sea surface temperature (SST) records from the southernmost Chilean fjord region $\left(50-53^{\circ} \mathrm{S}\right)$. SST estimates may be biased towards summer temperature in this region, as revealed by a large set of surface sediments. The Holocene records show consistently warmer than present-day SSTs except for the past $\sim 0.6$ cal ka BP. However, they do not exhibit an early Holocene temperature optimum as registered further north off Chile and in Antarctica. This may have resulted from a combination of factors including decreased inflow of warmer open marine waters due to lower sea-level stands, enhanced advection of colder and fresher inner fjord waters, and stronger westerly winds. During the midHolocene, pronounced short-term variations of up to $2.5^{\circ} \mathrm{C}$ and a cooling centered at $\sim 5 \mathrm{cal} \mathrm{ka}$ BP, which coincides with the first Neoglacial glacier advance in the Southern Andes, are recorded. The latest Holocene is characterized by two pronounced cold events centered at $\sim 0.6$ and 0.25 cal ka BP, i.e., during the Little Ice Age. These cold events have lower amplitudes in the offshore records, suggesting an amplification of the SST signal in the inner fjords. (c) 2014 University of Washington. Published by Elsevier Inc. All rights reserved.
\end{abstract}

\section{Introduction}

The Holocene (since 11.7 cal ka BP) has been traditionally considered as a period characterized by relatively stable climate conditions when compared to the last glacial period. However, substantial Holocene climate variability on millennial to centennial timescales has been reported mainly from the Northern Hemisphere (e.g., Mayewski et al., 2004; Wanner et al., 2008) while high-resolution Holocene temperature records from the Southern Hemisphere are still sparse (e.g., Kilian and Lamy, 2012). A recent effort to reconstruct a stacked temperature record from the latitudinal range $30^{\circ}-90^{\circ} \mathrm{S}$ includes, for example, only about ten marine and ice-core records (Marcott et al., 2013). Thus, more well-dated marine records from the Southern Hemisphere are needed.

Paleoclimate archives from Antarctica and the Southern Ocean reveal contrasting results pointing to a complex temperature evolution throughout the Holocene (e.g., Masson et al., 2000; Masson-Delmotte et al., 2004; Bentley et al., 2009; Divine et al., 2010; Shevenell et al., 2011). An early Holocene Climatic Optimum (11.5-9 cal ka BP) has

\footnotetext{
* Corresponding author at: Department of Oceanography and COPAS Sur-Austral Program, University of Concepción, Concepción, Chile.

E-mail address: mcaniupan@udec.cl (M. Caniupán).
}

been widely documented in Antarctic ice-core records, with temperatures up to $\sim 2^{\circ} \mathrm{C}$ warmer than present (e.g., Masson et al., 2000; WAIS Divide Project Members, 2013). Thereafter, results from ice cores suggest a long-term Antarctic cooling during the Holocene (MassonDelmotte et al., 2004). However, a secondary mid-Holocene temperature maximum between $\sim 8$ and $6 \mathrm{cal}$ ka BP appears to be important in the Ross Sea area whereas in eastern Antarctica some records show a weak secondary warming between $\sim 6$ and 3 cal ka BP (Masson et al., 2000). A recent $\mathrm{TEX}_{86}$-based SST record from the Palmer Deep at the continental margin of the western Antarctic Peninsula shows a cooling by $3-4^{\circ} \mathrm{C}$ over the past $12,000 \mathrm{yr}$ following the decline in local spring insolation (Shevenell et al., 2011). However, absolute SST values of this record appear to be low and doubts have been raised about the reliability of TEX ${ }_{86}$-based SST reconstructions in the Southern Ocean (Ho et al., 2014). Marine and lacustrine records around the Antarctic Peninsula confirm the complexity of the Holocene temperature evolution as maximum warming was registered during different time intervals in the early to middle Holocene (e.g., Bentley et al., 2009).

Marine records from the South Atlantic sector of the Southern Ocean $\left(50^{\circ}-53^{\circ} \mathrm{S}\right)$ reveal an early Holocene temperature optimum and the onset of cooling together with sea-ice expansion between $\sim 9$ and 7 cal ka BP (Bianchi and Gersonde, 2004; Nielsen et al., 2004). Earlier work in this region suggested an increase in the deposition of ice- 
rafted debris in the subantarctic South Atlantic starting abruptly at $\sim 5.5$ cal ka BP, which coincides with the onset of the Neoglacial phase and with an advance of sea-ice cover around Antarctica (Hodell et al., 2001; lizuka et al., 2008).

Holocene climate fluctuations in the Chilean fjord region $\left(\sim 42^{\circ}-\right.$ $55^{\circ} \mathrm{S}$ ), the closest land mass to Antarctica, are still not well documented due to the lack of records covering the complete Holocene with a high temporal resolution (Kilian and Lamy, 2012). In contrast, more detailed paleoceanographic reconstructions have been obtained from the Southeast Pacific off mid-latitude Chile $\left(\sim 30^{\circ}-41^{\circ} \mathrm{S}\right)$. At ODP Site $1233\left(41^{\circ} \mathrm{S}\right)$ located offshore the Northern Patagonia fjord region, warmest conditions occurred in the early Holocene ( 12-9 cal ka BP) (Kaiser et al., 2005); SSTs during this interval were generally $\sim 1-2^{\circ} \mathrm{C}$ above modern values. Thereafter, temperatures gradually declined reaching modern values $\left(\sim 14^{\circ} \mathrm{C}\right)$ in the late Holocene. The GeoB 3313-1 record (same location as ODP Site 1233; Lamy et al., 2002) covers the past $\sim 8$ cal ka BP and shows a secondary mid-Holocene warming at $6-5 \mathrm{cal} \mathrm{ka}$ BP and declining SSTs towards the late Holocene in a similar mode as the comparatively lower-resolution SST record from Site 1233. The recently published record from the Pacific entrance of the Strait of Magellan (Harada et al., 2013) shows warming in the early Holocene reaching a climate optimum from $\sim 11$ to $\sim 6$ cal ka BP but interrupted by a cool event at ca. 10.5 cal ka BP.

The early to mid-Holocene temperature maximum appears to coincide with widespread arid conditions onshore, as recorded in various terrestrial and marine records from central Chile $\left(32^{\circ}-35^{\circ} \mathrm{S}\right)$ (Villagrán, 1990; Lamy et al., 1999; Jenny et al., 2002; Maldonado and Villagrán, 2002; Villa-Martínez et al., 2003) and Northern Patagonia in Isla Grande de Chiloé (Abarzúa et al., 2004). It has been proposed that the more arid conditions on land resulted from a southward displacement of the southern westerly wind belt (SWW), which is the main source of precipitation (e.g., Kaiser et al., 2005). Offshore, changes within the Antarctic Circumpolar Current (ACC) might have diminished the intrusion of cold subantarctic waters into the Peru-Chile Current leading to a warming in the SSTs at least at the central Chilean margin. For the southernmost Chilean fjord region on the other hand, new precipitation reconstructions point to humid/windier conditions in the early Holocene, decreasing during the middle and late Holocene and suggesting an antiphase behavior between the core and the northern margin of the SWW (Lamy et al., 2010).

With the goal of filling the gap of paleoclimate information between high and mid-latitudes of southwestern South America, we provide new high-resolution records of SST variability throughout the complete Holocene in the Chilean fjord region (Churruca fjord at $\sim 53^{\circ} \mathrm{S}$, Canal Concepción at $\sim 51^{\circ} \mathrm{S}$, and Canal Wide at $\sim 50^{\circ} \mathrm{S}$ ). With the aim of describing regional patterns of paleoclimate changes and identify common forcing mechanisms, we compare our alkenone-derived SST data with previously published paleotemperature records from the Chilean margin and Northern Patagonian fjords between $\sim 41^{\circ}$ and $\sim 53^{\circ} \mathrm{S}$. Furthermore, in order to detect potential seasonality in the alkenone-derived SST signal, we analyzed a set of surface sediments distributed within the Chilean fjord region $\left(\sim 42^{\circ}-55^{\circ} \mathrm{S}\right)$.

\section{Study area}

Between $\sim 42^{\circ}$ and $55^{\circ} \mathrm{S}$, the Chilean continental margin is characterized by up to $200 \mathrm{~km}$ wide fjord belt, formed mainly by Pleistocene glacial erosion, that extends partially across the Andes Cordillera (Breuer et al., 2013). Over the last glacial termination, during the early stage of the Patagonian Ice Field retreat, up to $1100 \mathrm{~m}$ deep proglacial lakes were formed. The subsequent marine transgression across the shallow coastal shelf (today 50-80 m water depth) started after 14.3 cal ka BP at the western entrance of the Strait of Magellan (Kilian et al., 2007). The Chilean fjord region intersect the core of the SWW with maximum precipitation of westerly origin that is recorded between $\sim 50^{\circ}$ and $55^{\circ} \mathrm{S}$ and diminishing northward and southward of these latitudes (e.g., Schneider et al., 2003; Garreaud et al., 2013). Modern oceanic surface circulation is dominated by the northern boundary of the ACC that bifurcates at $\sim 45^{\circ} \mathrm{S}$ into the Peru-Chile Current (PCC) flowing equatorward and the Cape Horn Current (CHC) flowing southward, both transporting Pacific subantarctic waters (SAAW) (e.g., Strub et al., 1998; Chaigneau and Pizarro, 2005; Sievers and Silva, 2008) (Fig. 1). The $\mathrm{CHC}$ transports anomalously warmer waters $\left(\sim 1.5^{\circ} \mathrm{C}\right)$ compared to subantarctic water masses in the open Pacific. At the same latitude, SSTs are similar to air temperatures during winter, whereas in spring and summer they are comparatively lower due to the input of fresh and cold water from snow and glacier melting (Kilian et al., 2013). The inner fjord system is characterized by a 30- to 70-m thick freshwater layer (salinity of 10-27 psu) derived from the very high regional precipitation ( 4000 to $>10,000 \mathrm{~mm} / \mathrm{yr}$, Schneider et al., 2003). Since the lowdensity of the surface cold freshwater layer strongly hampers mixing with warmer fjord subsurface water (Kilian et al., 2007), the summer fjord SST's are often $2-4^{\circ} \mathrm{C}$ lower than local air temperatures (Kilian et al., 2013). The westward expansion of this freshwater layer towards the Pacific is additionally controlled by the SWW strength. Relatively warmer SAAW, with salinities of $>32$ psu, enters the fjord system below the freshwater layer and forms a dense and thus stable bottom water body (e.g., Sievers and Silva, 2008). Thus, fjord freshwaters and Pacific SAAW constitute a two-layer estuarine circulation, with fresh waters flowing towards the ocean at the surface and saltier Pacific SAAW into the fjords at depth (e.g., Sievers and Silva, 2008). The three coring sites studied here are in different oceanographic and continental settings. Canal Concepción is characterized by a strong marine influence, while Canal Wide is under the strong influence of glacier meltwater as it is located close to the Southern Patagonian Ice Field. The Churruca fjord, which is located within the Magellan fjord system, receives a high amount of freshwater from annual precipitation and glacier melting in austral spring.

Annual mean SST (World Ocean Atlas 2009 (WOA09); Locarnini et al., 2010) increases from $8^{\circ} \mathrm{C}$ at $\sim 52^{\circ} \mathrm{S}$ to $13^{\circ} \mathrm{C}$ at $\sim 42^{\circ} \mathrm{S}$ in the oceanic area adjacent to the Chilean fjords (Fig. 1). For the Northern Patagonian fjords, in-situ SST measurements have been performed during several Chilean cruises in austral spring, summer and/or winter since 1995 within the CIMAR Program (Cruceros de Investigación Marina en Áreas Remotas) (e.g., Silva et al., 1997; Silva and Calvete, 2002) whereas further south only austral spring data are available within this program (http://www.shoa.cl/n_cendhoc/index.html). Data from individual cruises as well as from an environmental monitoring network at $52^{\circ}-53^{\circ} \mathrm{S}$ have been added to the dataset from the southernmost fjords (Kilian et al., 2013). Compared to SST data in the adjacent open ocean, temperatures in the fjords are generally colder due to the high contribution of estuarine waters, the influence of colder air temperatures inland and high meteoric precipitation reaching up to $10 \mathrm{~m} / \mathrm{yr}$ in western Patagonia (e.g., Schneider et al., 2003).

\section{Materials and methods}

The alkenone-derived SST records from the Chilean fjord region include core MD07-3124 (22.25 m) from Canal Concepción (50³0.96'S; $74^{\circ} 58.33^{\prime} \mathrm{W}$; $564 \mathrm{~m}$ water depth) collected during the XV-MD-159PACHIDERME Expedition on board R/V Marion Dufresne; core JPC-42 $(12.5 \mathrm{~m})$ recovered between the Europa and Penguin fjords in the Canal Wide $\left(49^{\circ} 55^{\prime} \mathrm{S} ; 74^{\circ} 23^{\prime} \mathrm{W}\right.$; $904 \mathrm{~m}$ water depth) during the NBP05-05 Palmer Expedition with RV/IB N.B. Palmer; and core CHURR which was retrieved from the Churruca fjord $\left(53^{\circ} 02^{\prime} \mathrm{S} ; 73^{\circ} 54^{\prime} \mathrm{W}\right.$; $80 \mathrm{~m}$ water depth; $11.2 \mathrm{~m}$ core length) in the western basin of the Strait of Magellan, on board R/V Gran Campo II. Core MD07-3124 was sampled at 8-cm intervals (corresponding to a mean temporal resolution of $\sim 100 \mathrm{yr}$ ); core JPC-42 was sampled at 10 -cm intervals (mean resolution of $\sim 110 \mathrm{yr}$ ); and core CHURR was sampled at 20-cm intervals (mean resolution of $\sim 200 \mathrm{yr}$ ). 


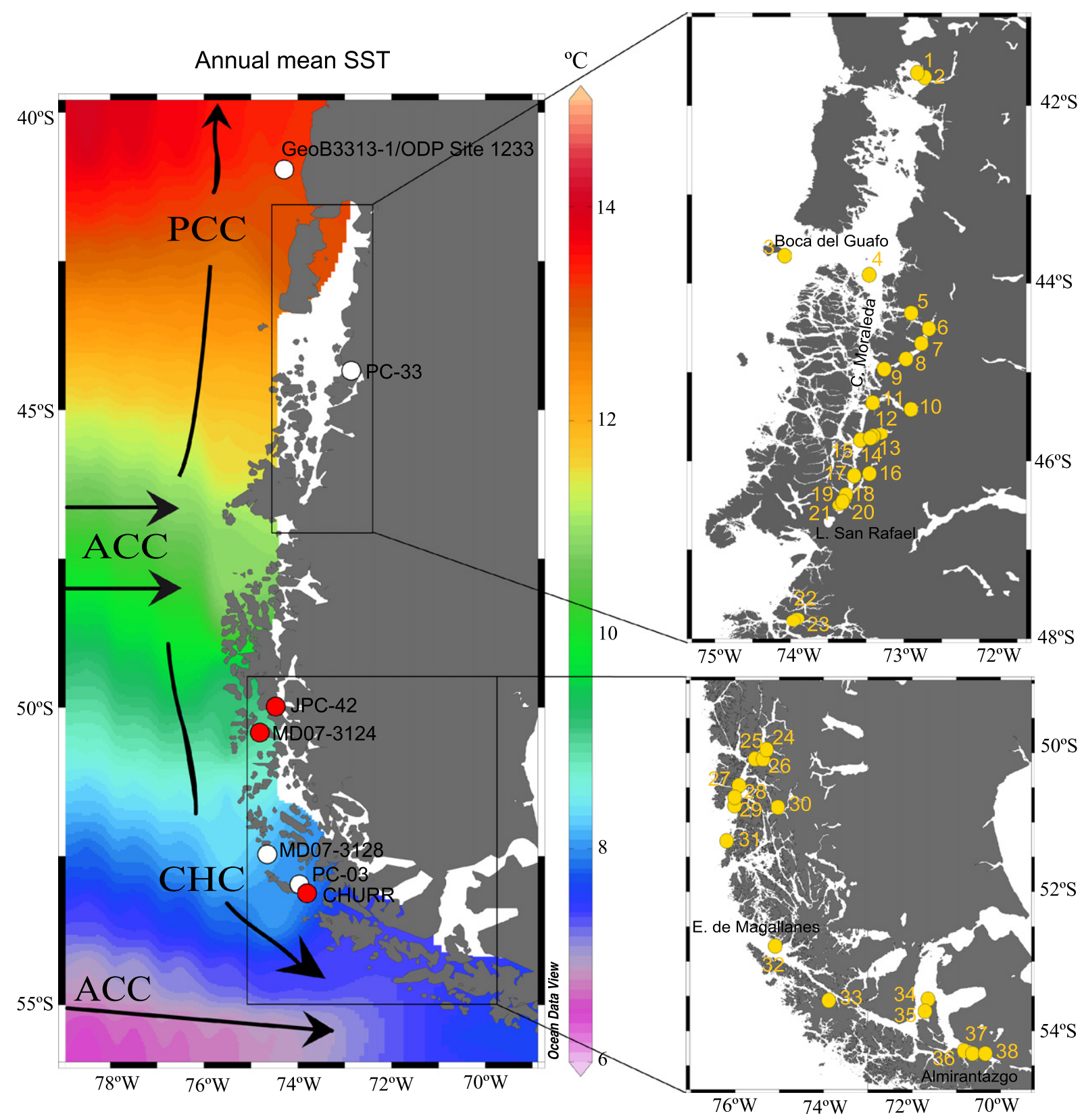

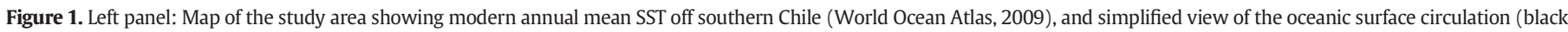

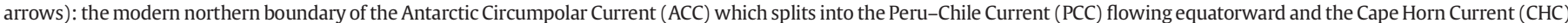

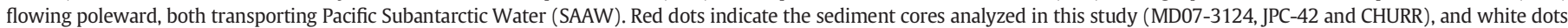

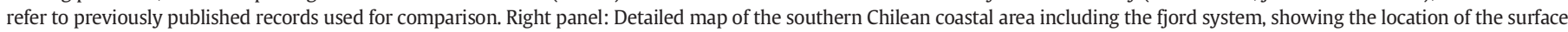
sediments used in this study (yellow dots; Table 1).

In addition to the sediment cores, alkenone-derived SSTs were also estimated from 38 surface sediment samples collected from $41^{\circ}$ to $55^{\circ} \mathrm{S}$ (Fig. 1) during various expeditions with coring devices that kept the water-sediment interface undisturbed. These include: 14 samples collected during the Chilean CIMAR FIORDO (CF) 7 Expedition; ten samples collected during the NBP05-05 Palmer Cruise; two samples collected during the Japanese BEAGLE expedition on board R/V Mirai; eight samples collected during the XV-MD-159-PACHIDERME cruise; and four samples taken during the R/V Gran Campo II cruise (Table 1).

\section{Chronology}

The chronology of core MD07-3124 is based on 15 AMS radiocarbon ages $\left({ }^{14} \mathrm{C}\right.$ ) (Table 2; Fig. 2) performed on mixed benthic carbonates (scaphopods, bivalves and benthic foraminifera) at the LeibnizLaboratory for Radiometric Dating and Isotope Research at the University of Kiel. The chronology of core JPC-42 includes six AMS- ${ }^{14} \mathrm{C}$ ages from shell fragments and one age from a piece of wood dated at the WHOI-NOSAMS facility (Table 2; Fig. 2). For core CHURR, the age model was constrained by eleven AMS- ${ }^{14} \mathrm{C}$ dates of molluscs and wood performed at the WHOI-NOSAMS facility and at the Department of Earth Systems Sciences, University of California Irvine. The radiometric chronology was supplemented by the occurrence of the well-dated 4.15 cal ka BP Mt. Burney tephra layer (McCulloch and Davies, 2001; Stern, 2008) (Table 2; Fig. 2).

Within JPC-42, the comparison of two AMS- ${ }^{14} \mathrm{C}$ dates, one from carbonate and the other from wood fragments obtained from the same core depth $(1.75 \mathrm{~m})$ suggests a marine reservoir age of $780 \mathrm{yr}$ 
Table 1

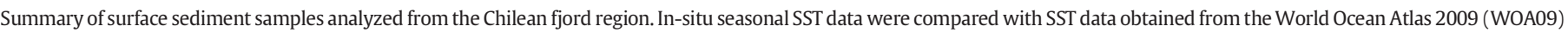

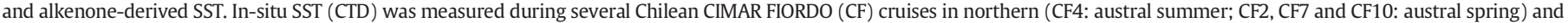
southern Patagonia (CF3: austral spring). In-situ and WOA temperatures refer to $10 \mathrm{~m}$ water depth.

\begin{tabular}{|c|c|c|c|c|c|c|c|c|c|c|}
\hline $\mathrm{N}^{\circ}$ & Description & $\begin{array}{l}\text { Lat } \\
\left({ }^{\circ} \mathrm{S}\right)\end{array}$ & $\begin{array}{l}\text { Long } \\
\left({ }^{\circ} \mathrm{W}\right)\end{array}$ & $\begin{array}{l}\text { Water depth } \\
(\mathrm{m})\end{array}$ & $\begin{array}{l}\text { Surface sediment } \\
\text { sampled }\end{array}$ & $\begin{array}{l}\text { Spring SST } \\
\text { in-situ/WOA }\left({ }^{\circ} \mathrm{C}\right)\end{array}$ & $\begin{array}{l}\text { Summer SST } \\
\text { in-situ/WOA }\left({ }^{\circ} \mathrm{C}\right)\end{array}$ & $\begin{array}{l}\text { Annual SST } \\
\text { WOA }\left({ }^{\circ} \mathrm{C}\right)\end{array}$ & $\begin{array}{l}\mathrm{U}^{\mathrm{K}^{\prime}} \\
\text { value }\end{array}$ & $\begin{array}{l}\text { Alk-SST } \\
\left({ }^{\circ} \mathrm{C}\right)\end{array}$ \\
\hline \multicolumn{11}{|c|}{ Northern Patagonia } \\
\hline 1 & MD07-3108 & 41.7 & 72.67 & 548 & Summer & 11.6/13.1 & $15.0 / 15.1$ & 12.9 & 0.456 & 12.3 \\
\hline 2 & $\begin{array}{l}\text { MD07-3105 } \\
\text { Boca del Guafo }\end{array}$ & 41.71 & 72.78 & 329 & Summer & 11.6/13.1 & $15.0 / 15.1$ & 12.9 & 0.422 & 11.3 \\
\hline 3 & $\begin{array}{l}\text { CF7-BC01 } \\
\text { Canal Moraleda }\end{array}$ & 43.73 & 74.58 & 240 & Spring & $11.3 / 12.2$ & $13.8 / 14.8$ & 12.3 & 0.430 & 11.5 \\
\hline 4 & $\begin{array}{l}\text { CF7-BC06 } \\
\text { Jacaf fjord }\end{array}$ & 43.99 & 73.36 & 176 & Spring & $11.9 / 12.2$ & $12.7 / 14.8$ & 12.3 & 0.421 & 11.2 \\
\hline 5 & $\begin{array}{l}\text { PC-33 } \\
\text { Canal Puyuguapi }\end{array}$ & 44.33 & 72.97 & 510 & Spring & $10.2 / 11.7$ & $/ 14.1$ & 11.8 & 0.384 & 10.1 \\
\hline 6 & CF7-BC36 & 44.44 & 72.62 & 219 & Spring & $11.7 / 11.7$ & $13.0 / 14.1$ & 11.8 & 0.411 & 10.9 \\
\hline 7 & CF7-BC39 & 44.73 & 72.71 & 160 & Spring & $11.7 / 11.7$ & $13.0 / 14.1$ & 11.8 & 0.403 & 10.7 \\
\hline 8 & CF7-BC40 & 44.82 & 72.93 & 260 & Spring & $11.7 / 11.7$ & $13.0 / 14.1$ & 11.8 & 0.412 & 11.0 \\
\hline 9 & $\begin{array}{l}\text { CF7-BC42 } \\
\text { Aysén fjord }\end{array}$ & 44.91 & 73.32 & 320 & Spring & 11.6/11.7 & $13.0 / 14.1$ & 11.8 & 0.430 & 11.5 \\
\hline 10 & CF7-BC17A & 45.36 & 73.29 & 330 & Spring & $10.4 / 11.4$ & $12.2 / 13.7$ & 11.5 & 0.424 & 11.3 \\
\hline 11 & $\begin{array}{l}\text { MD07-3114 } \\
\text { Estero Quitralco }\end{array}$ & 45.38 & 73.47 & 294 & Summer & $10.4 / 11.4$ & $12.2 / 13.7$ & 11.5 & 0.423 & 11.3 \\
\hline 12 & CF7-BC30 & 45.74 & 73.41 & 269 & Spring & $10.3 / 11.4$ & $12.2 / 13.7$ & 11.5 & 0.430 & 11.5 \\
\hline 13 & CF7-BC30A & 45.75 & 73.51 & 110 & Spring & $10.1 / 11.4$ & $12.2 / 13.7$ & 11.5 & 0.406 & 10.8 \\
\hline 14 & CF7-BC29A & 45.76 & 73.47 & 112 & Spring & $10.2 / 11.4$ & $11.9 / 13.7$ & 11.5 & 0.390 & 10.3 \\
\hline 15 & $\begin{array}{l}\text { CF7-BC29 } \\
\text { Estero Cupquelán }\end{array}$ & 45.78 & 73.51 & 114 & Spring & $10.2 / 11.4$ & $11.9 / 13.7$ & 11.5 & 0.402 & 10.7 \\
\hline 16 & $\begin{array}{l}\text { CF7-BC28 } \\
\text { Laguna San Rafael }\end{array}$ & 46.14 & 73.49 & 239 & Spring & $9.6 / 10.5$ & $11.5 / 13.3$ & 10.9 & n.d. & \\
\hline 17 & Palmer-KC03 & 46.16 & 73.66 & 52 & Winter & $8.8 / 10.5$ & $11.0 / 13.3$ & 10.9 & n.d. & \\
\hline 18 & Palmer-KC13 & 46.44 & 73.79 & 30 & Winter & $8.8 / 10.5$ & $11.0 / 13.3$ & 10.9 & n.d. & \\
\hline 19 & Palmer-KC15 & 46.43 & 73.79 & 112 & Winter & $8.8 / 10.5$ & $11.0 / 13.3$ & 10.9 & n.d. & \\
\hline 20 & Palmer-KC16 & 46.43 & 73.80 & 112 & Winter & $8.8 / 10.5$ & $11.0 / 13.3$ & 10.9 & n.d. & \\
\hline 21 & $\begin{array}{l}\text { CF7-BC27 } \\
\text { Seno Baker }\end{array}$ & 46.48 & 73.80 & 112 & Spring & $8.8 / 10.5$ & $11.0 / 13.3$ & 10.9 & n.d. & \\
\hline 22 & MD07-3120 & 47.88 & 74.49 & 662 & Summer & $9.5 / 10.7$ & $11.9 / 13.0$ & 10.9 & 0.452 & 12.1 \\
\hline 23 & MD07-3122 & 47.88 & 74.49 & 663 & Summer & $9.5 / 10.7$ & 11.9/13.0 & 10.9 & 0.452 & 12.1 \\
\hline Sou & $\begin{array}{l}\text { rn Patagonia } \\
\text { Penguin fjord }\end{array}$ & & & & & & & & & \\
\hline 24 & $\begin{array}{l}\text { Palmer-KC } 41 \\
\text { Europa fjord }\end{array}$ & 49.91 & 74.38 & 711 & Winter & $6.6 / 8.6$ & $10.8 / 11.3$ & 9.2 & 0.368 & 9.7 \\
\hline 25 & Palmer-KC29 & 50.01 & 74.40 & 350 & Winter & $6.7 / 8.6$ & $10.8 / 11.3$ & 9.2 & 0.366 & 9.6 \\
\hline 26 & $\begin{array}{l}\text { Palmer-KC27 } \\
\text { Canal Concepción }\end{array}$ & 50.06 & 74.43 & 414 & Winter & $6.7 / 8.6$ & $10.8 / 11.3$ & 9.2 & 0.369 & 9.7 \\
\hline 27 & MD07-3123 & 50.5 & 74.97 & 567 & Summer & $7.6 / 8.6$ & $11.4 / 11.3$ & 9.2 & 0.410 & 10.9 \\
\hline 28 & MD07-3124 & 50.51 & 74.98 & 564 & Summer & $7.6 / 8.6$ & $11.4 / 11.3$ & 9.2 & 0.392 & 10.4 \\
\hline 29 & $\begin{array}{l}\text { Conce-1SL } \\
\text { Peel fjord }\end{array}$ & 50.6 & 74.98 & 191 & Spring & $7.6 / 8.6$ & $11.4 / 11.3$ & 9.2 & 0.44 & 11.8 \\
\hline 30 & $\begin{array}{l}\text { Peel-4SL } \\
\text { Jorge Montt Island }\end{array}$ & 50.81 & 74.0 & 39 & Spring & $6.2 / 8.6$ & $/ 11.3$ & 9.2 & 0.300 & 7.7 \\
\hline 31 & $\begin{array}{l}\text { DDA-1SL } \\
\text { E. de Magallanes }\end{array}$ & 51.25 & 75.1 & 215 & Spring & $8.5 / 8.6$ & $/ 10.1$ & 8.2 & 0.360 & 9.4 \\
\hline 32 & Beagle-MC03 & 52.87 & 74.09 & 528 & Spring & $7.0 / 7.9$ & $10.4 / 9.8$ & 8.2 & 0.395 & 10.5 \\
\hline 33 & FART SL & 53.46 & 72.8 & & Spring & $6.6 / 7.7$ & /9.2 & 7.8 & 0.310 & 8.0 \\
\hline 34 & Beagle-MC04 & 53.57 & 70.67 & 428 & Spring & $6.6 / 7.7$ & 8.6/9.2 & 7.8 & 0.354 & 9.3 \\
\hline 35 & $\begin{array}{l}\text { MD07-3131 } \\
\text { Seno Almirantazgo }\end{array}$ & 53.57 & 70.67 & 463 & Summer & $6.6 / 7.7$ & $8.6 / 9.2$ & 7.8 & 0.331 & 8.6 \\
\hline 36 & Palmer-KC76 & 54.26 & 69.79 & 290 & Winter & $6.1 / 7.0$ & $8.2 / 8.4$ & 7.2 & 0.345 & 9.0 \\
\hline 37 & Palmer-KC66 & 54.32 & 69.46 & 110 & Winter & $5.8 / 7.0$ & $8.2 / 8.4$ & 7.2 & 0.331 & 8.6 \\
\hline 38 & Palmer-KC68 & 54.32 & 69.55 & 117 & Winter & $5.8 / 7.0$ & $8.2 / 8.4$ & 7.2 & 0.330 & 8.6 \\
\hline
\end{tabular}

n.d. $=$ not detected.

$(\triangle \mathrm{R} \sim 400 \mathrm{yr})$ at $0.76 \mathrm{cal} \mathrm{ka} \mathrm{BP}$, if we assume the marine and terrestrial samples were contemporaneous (Table 2). Although this reservoir age is greater than the published age of $530 \mathrm{yr}$ from the Puerto Natales region (Ingram and Southon, 1996), new surface reservoir-age data from the southern Chilean margin agree with our estimate (ca. $800 \mathrm{yr}$ in core MD07-3088 at $46^{\circ} \mathrm{S}$; Siani et al., 2013); other records from many fjord settings have suggested relatively large reservoir corrections (e.g., Milliken et al., 2009). Though we are primarily dating benthic carbonate, we do not expect a substantial increase of reservoir ages in the fjord basin (sites MD07-3124 and JPC-42) as these are filled with more saline surface water from the open Pacific due to the shallow sill depths at the entrance channels. For core CHURR, however, we applied a marine reservoir age of $500 \mathrm{yr}$ estimated by comparing sedimentation rates between sections with the Mt. Burney tephra and dated tree wood fragments. The lower reservoir age at the CHURR site can be related to the fact that this core was collected from very shallow water depth under the influence of shallower, fresher surface waters. Accordingly, radiocarbon ages from MD07-3124 and JPC-42 cores were corrected for a marine reservoir of $780 \mathrm{yr}$ and those from CHURR core for $500 \mathrm{yr}$ and calibrated to calendar thousand years before present (cal ka BP) 
Table 2

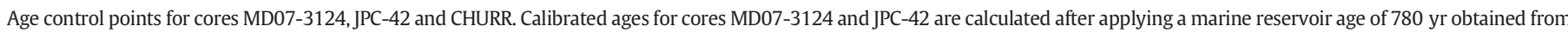

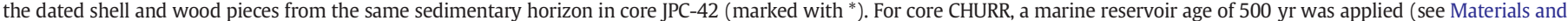
methods).

\begin{tabular}{|c|c|c|c|c|c|c|}
\hline $\begin{array}{l}\text { Core depth } \\
\text { (meters) }\end{array}$ & Lab. code & $\begin{array}{l}\text { Dated } \\
\text { material }\end{array}$ & $\begin{array}{l}\text { Reservoir } \\
\text { age (yr) }\end{array}$ & $\begin{array}{l}\text { Conventional age } \\
\left({ }^{14} \mathrm{C} \text { yr BP }\right) \pm \text { error }\end{array}$ & $\begin{array}{l}\text { Calibrated age (cal yr BP) } \\
2 \sigma \text { range, probability }\end{array}$ & $\begin{array}{l}\text { Calibrated age final } \\
\text { (cal yr BP) } 2 \sigma, \pm \text { error }\end{array}$ \\
\hline & MD07-3124 & & & & & \\
\hline 0.65 & WHOI 69341 & Shell & 780 & $1110 \pm 30$ & $308-472(1)$ & $390 \pm 82$ \\
\hline 1.25 & WHOI 69342 & Shell & 780 & $1690 \pm 30$ & $760-916(0.986)$ & $839 \pm 79$ \\
\hline 2.30 & WHOI 69343 & Shell & 780 & $2830 \pm 35$ & $1926-2118(1)$ & $2022 \pm 96$ \\
\hline \multirow[t]{3}{*}{3.10} & KIA 33278 & Shell & 780 & $3295 \pm 30$ & $2490-2643(0.70)$ & $2605 \pm 126$ \\
\hline & & & & & $2654-2667(0.02)$ & \\
\hline & & & & & $2676-2741(0.28)$ & \\
\hline 4.30 & WHOI 69344 & Shell & 780 & $4230 \pm 40$ & $3614-3832(1)$ & $3723 \pm 109$ \\
\hline 5.60 & WHOI 69345 & Shell & 780 & $5280 \pm 35$ & 5039-5303 (1) & $5171 \pm 132$ \\
\hline \multirow[t]{3}{*}{6.65} & WHOI 69346 & Shell & 780 & $6050 \pm 30$ & $5940-5977(0.14)$ & $6051 \pm 120$ \\
\hline & & & & & $5981-6124(0.69)$ & \\
\hline & & & & & $6144-6179(0.17)$ & \\
\hline 7.0 & WHOI 69347 & Shell & 780 & $6380 \pm 40$ & $6299-6453(1)$ & $6376 \pm 77$ \\
\hline \multirow[t]{3}{*}{7.55} & KIA 33279 & Shell & 780 & $6850 \pm 35$ & $6797-6819(0.04)$ & $6913 \pm 174$ \\
\hline & & & & & $6831-7011(0.94)$ & \\
\hline & & & & & $7129-7144(0.02)$ & \\
\hline 10.30 & WHOI 69348 & Shell & 780 & $8040 \pm 60$ & $7968-8180(1)$ & $8074 \pm 106$ \\
\hline 13.83 & WHOI 69349 & Shell & 780 & $8840 \pm 55$ & $8747-9126(0.99)$ & $8937 \pm 190$ \\
\hline \multirow[t]{2}{*}{15.38} & KIA 33280 & Shell & 780 & $9090 \pm 45$ & $9139-9179(0.07)$ & $9314 \pm 156$ \\
\hline & & & & & $9200-9450(0.93)$ & \\
\hline 16.8 & WHOI 69350 & Shell & 780 & $9360 \pm 45$ & $9484-9631(0.99)$ & $9558 \pm 74$ \\
\hline \multirow[t]{2}{*}{19.62} & KIA 33281 & Shell & 780 & $9880 \pm 45$ & $10192-10303(0.85)$ & $10263 \pm 100$ \\
\hline & & & & & $10313-10390(0.15)$ & \\
\hline \multirow[t]{4}{*}{22.10} & WHOI 69351 & Shell & 780 & $10250 \pm 50$ & $10574-10819(0.81)$ & $10754 \pm 248$ \\
\hline & & & & & $10846-10866(0.02)$ & \\
\hline & & & & & $10953-11069(0.17)$ & \\
\hline & JPC-42 & & & & & \\
\hline \multirow[t]{3}{*}{0.74} & WHOI-35 50330 & Shell & 780 & $995 \pm 90$ & $0-48(0.12)$ & $212 \pm 229$ \\
\hline & & & & & $53-336(0.70)$ & \\
\hline & & & & & $348-458(0.18)$ & \\
\hline \multirow[t]{2}{*}{1.23} & WHOI-36 50326 & Shell & 780 & $1150 \pm 35$ & $316-406(0.45)$ & $416 \pm 94$ \\
\hline & & & & & $421-503(0.55)$ & \\
\hline 1.75 & WHOI-38 50328 & Shell* & 780 & $1620 \pm 90$ & $655-932(1)$ & $794 \pm 139$ \\
\hline 1.75 & WHOI-37 50327 & Wood* & & $840 \pm 75$ & $675-915(1)$ & $794 \pm 122$ \\
\hline \multirow[t]{2}{*}{6.55} & WHOI-39 50329 & Shell & 780 & $7370 \pm 40$ & $7430-7523(0.80)$ & $7491 \pm 68$ \\
\hline & & & & & $7531-7565(0.20)$ & \\
\hline \multirow[t]{4}{*}{8.46} & WHOI-40 50331 & Shell & 780 & $8620 \pm 65$ & $8454-8794(0.89)$ & $8654 \pm 261$ \\
\hline & & & & & $8828-8867(0.04)$ & \\
\hline & & & & & $8882-8899(0.01)$ & \\
\hline & & & & & $8911-8975(0.06)$ & \\
\hline \multirow[t]{3}{*}{11.48} & WHOI-41 50324 & Shell & 780 & $10900 \pm 60$ & $11404-11579(0.17)$ & $11745 \pm 301$ \\
\hline & & & & & $11587-12005(0.83)$ & \\
\hline & CHURR & & & & & \\
\hline 0.58 & UCIAMS 82045 & Molluscs & 500 & $1325 \pm 15$ & $691-767(1)$ & $729 \pm 38$ \\
\hline \multirow[t]{2}{*}{0.78} & UCIAMS 82046 & Molluscs & 500 & $1500 \pm 20$ & $831-850(0.07)$ & $925 \pm 65$ \\
\hline & & & & & $906-961(0.93)$ & \\
\hline 1.38 & UCIAMS 82047 & Molluscs & 500 & $2070 \pm 15$ & $1412-1522(1)$ & $1467 \pm 55$ \\
\hline \multirow[t]{3}{*}{2.60} & UCIAMS 82048 & Molluscs & 500 & $3275 \pm 20$ & $2795-2832(0.15)$ & $2873 \pm 74$ \\
\hline & & & & & $2836-2929(0.84)$ & \\
\hline & & & & & $2935-2942(0.02)$ & \\
\hline 3.13 & UCIAMS 82049 & Molluscs & 500 & $3730 \pm 15$ & $3398-3477$ (1) & $3438 \pm 40$ \\
\hline 3.54 & UCIAMS 82050 & Molluscs & 500 & $4205 \pm 15$ & $3983-4090(1)$ & $4037 \pm 54$ \\
\hline 3.69 & & Tephra & & & 4150 & 4150 \\
\hline \multirow[t]{2}{*}{5.31} & UCIAMS 82051 & Molluscs & 500 & $5595 \pm 25$ & $5749-5829(0.65)$ & $5822 \pm 82$ \\
\hline & & & & & $5855-5913(0.35)$ & \\
\hline 6.27 & UCIAMS 82052 & Molluscs & 500 & $6935 \pm 25$ & $7308-7425(1)$ & $7367 \pm 117$ \\
\hline 8.03 & UCIAMS 74687 & Wood & & $8160 \pm 35$ & $9011-9144(0.82)$ & $9101 \pm 120$ \\
\hline & & & & & $9167-9251(0.18)$ & \\
\hline 9.03 & UCIAMS 74688 & Wood & & $8355 \pm 20$ & $9303-9364(0.41)$ & $9380 \pm 76$ \\
\hline & & & & & $9370-9456(0.59)$ & \\
\hline 10.80 & UCIAMS 74689 & Wood & & $9740 \pm 50$ & $10884-10926(0.04)$ & $11153 \pm 181$ \\
\hline & & & & & $11083-11246(0.96)$ & \\
\hline
\end{tabular}

using the Calib Rev 7.0 and IntCal13 software (Reimer et al., 2013) (Table 2).

Alkenone-derived sea-surface temperature (SST)

Alkenone analyses of the surface sediments and cores MD07-3124, JPC-42 and CHURR were carried out following the procedure described by Müller et al. (1998). Lipids were extracted from 3 to $5 \mathrm{~g}$ of homogenized and freeze-dried sediment by sonication $(3 \times)$ using successively less polar mixtures of methanol and dichloromethane. The combined extracts were washed with deionized water, dried over sodium sulfate and concentrated to dryness with a rotary evaporator. Extracts were saponified to avoid interferences with co-eluting $C_{36}$-fatty acid methyl esters. The fraction containing the alkenones was purified using a silica gel 


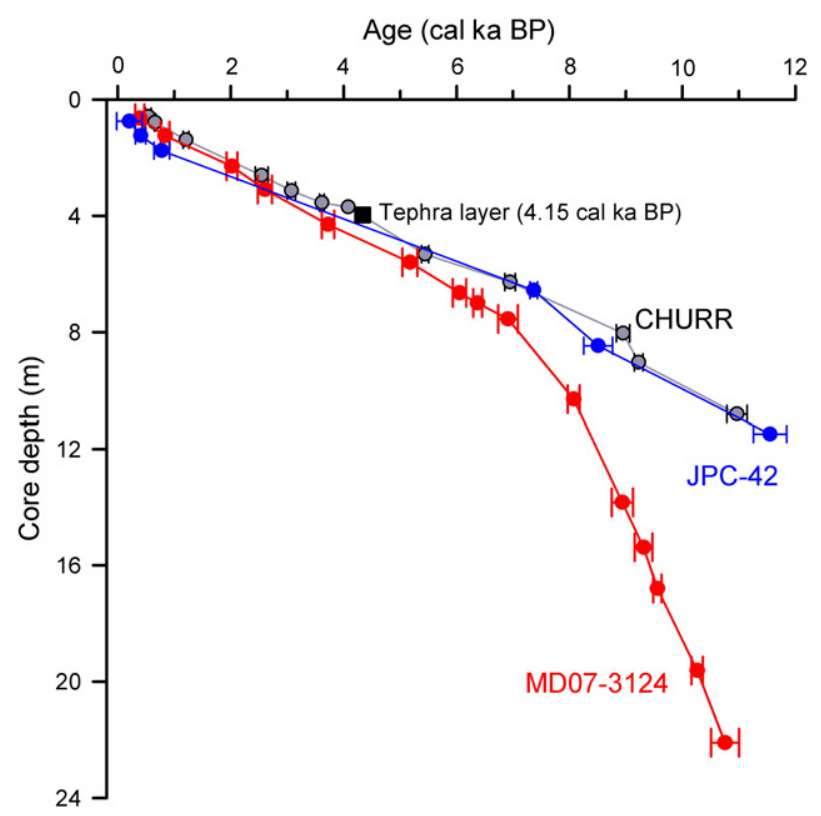

Figure 2. Age-depth relationship for cores MD07-3124, JPC-42 and CHURR based on calibrated ${ }^{14} \mathrm{C}$ ages (cal yr BP). Horizontal bars represent the standard deviation of measurements. Black square on CHURR core corresponds to the occurrence of the Mt. Burney tephra layer. See Table 2 for data.

cartridge. Identification and quantification of di- and triunsaturated $\mathrm{C}_{37}$-alkenones were achieved by gas chromatography on a $60 \mathrm{~m}$ fused silica capillary column (DB-5 MS, Agilent) with a flame ionization detector. The oven temperature was programmed to rise from $50^{\circ}$ to $250^{\circ} \mathrm{C}$ at $25^{\circ} \mathrm{C} / \mathrm{min}$, then to $290^{\circ} \mathrm{C}$ at $1{ }^{\circ} \mathrm{C} / \mathrm{min}$, held for $26 \mathrm{~min}$, and to $310^{\circ} \mathrm{C}$ at $30^{\circ} \mathrm{C} / \mathrm{min}$ and held constant for $10 \mathrm{~min}$. Alkenone measurements on core MD07-3124 were performed at the University of Bremen and measurements on cores JPC-42 and CHURR at the University of Concepción. Measurements on surface sediment samples were conducted partly at the University of Concepción and partly at the Leibniz Institute for Baltic Sea Research. For all samples, we used the simplified alkenone unsaturation index $\mathrm{U}^{\mathrm{K}^{\prime}}{ }_{37}=\left(\mathrm{C}_{37: 2}\right) /\left(\mathrm{C}_{37: 2}+\mathrm{C}_{37: 3}\right)$ (Prahl and
Wakeham, 1987). Triplicate analysis on four surface sediment samples shows a standard deviation of 0.018 units for the $\mathrm{U}^{\mathrm{K}^{\prime}}{ }_{37}$ measurements, corresponding to an error of $\pm 0.5^{\circ} \mathrm{C}$ in temperature estimates. In order to compare our new SST reconstructions with previously published temperature estimates from the Southeast Pacific (Lamy et al., 2002; Kaiser et al., 2005; Harada et al., 2013), we have used the culture calibration from Prahl et al. (1988); $\left(\mathrm{U}^{\mathrm{K}^{\prime}} 37=0.034 \mathrm{~T}+0.039\right)$ since it is similar to both global core top calibrations (Müller et al., 1998; Conte et al., 2006).

\section{Results}

\section{SST distribution and alkenone-derived SST from surface sediments}

We compare our alkenone-derived SST estimates from surface sediments collected in the Chilean fjord region to the in-situ SST data (mainly from CTDs as part of the CIMAR FIORDO program of the Chilean Navy) and to the WOA09 SST data for austral spring and summer (Table 1), in order to identify the seasonal signal in the alkenone-derived SST.

Inside the fjords, in-situ austral spring and summer SSTs decrease with latitude (Table 1, Fig. 3). Overall, in-situ spring SSTs are $\sim 2-3^{\circ} \mathrm{C}$ colder than in-situ summer SST; this seasonal difference is even more pronounced in those fjords located near glaciers (i.e. Penguin and Europa fjords; Fig. 1). Compared to seasonal SST data offshore (WOA09), spring and summer temperatures inside the fjords are generally colder by $\sim 1^{\circ} \mathrm{C}$ (Fig. 3 ).

Alkenone-derived SSTs were quantified in 33 out of the 38 surface sediment samples analyzed from the Chilean fjord region (Fig. 1; Table 1). No alkenone signal was detected near Laguna San Rafael at $46.5^{\circ} \mathrm{S}$, an area that is presently under strong influence of glacier meltwater with salinities between 22.2 and 23.2 psu (Silva et al., 1998). In Northern Patagonia $\left(44^{\circ}-48^{\circ} \mathrm{S}\right)$, the mean reconstructed SST value is $11^{\circ} \mathrm{C}$ with higher temperatures at stations with comparatively strong open marine influence (i.e. Boca del Guafo $\left(11.5^{\circ} \mathrm{C}\right)$, entrance of Canal Moraleda $\left(11.2^{\circ} \mathrm{C}\right)$, mouth of Canal Puyuhuapi $\left(11.5^{\circ} \mathrm{C}\right)$, and Seno Baker $\left(12.1^{\circ} \mathrm{C}\right)$ ) (Table 1; Figs. 1 and 3). SSTs diminish in inner fjords (i.e., Jacaf fjord $\left(10.1^{\circ} \mathrm{C}\right)$, head of Canal Puyuhuapi $\left(10.7^{\circ} \mathrm{C}\right)$ and Estero Quitralco $\left(10.3^{\circ} \mathrm{C}\right)$ ). Compared to seasonal in-situ SST data, the reconstructed SST for Northern Patagonia largely corresponds to austral

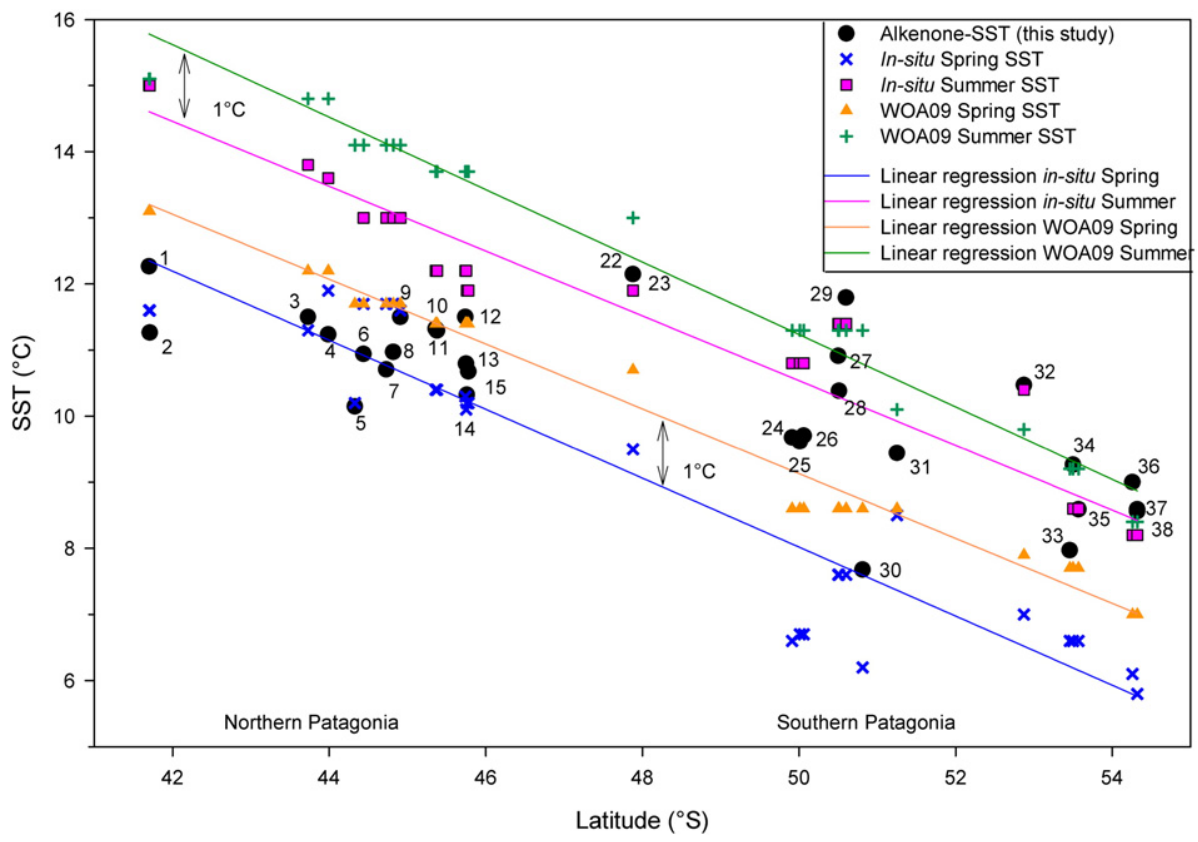

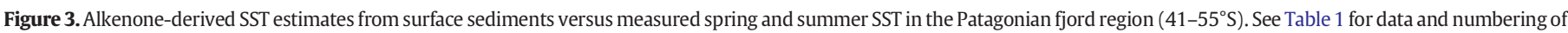
surface sediment samples. 
spring temperatures (Table 1, Fig. 3). In Southern Patagonia $\left(48^{\circ}-54^{\circ} \mathrm{S}\right)$, the mean reconstructed SST is $9^{\circ} \mathrm{C}$. The highest reconstructed SSTs were obtained in samples located close to the open Pacific (i.e., Canal Concepción $\left(11.8^{\circ} \mathrm{C}\right)$ and the Pacific entrance of the Strait of Magellan $\left(10.5^{\circ} \mathrm{C}\right)$ ), and the coldest SSTs were obtained inside the fjords (i.e., in the Peel $\left(7.7^{\circ} \mathrm{C}\right)$ and Europa $\left(8.3^{\circ} \mathrm{C}\right)$ fjords at $\sim 50^{\circ} \mathrm{S}$, in the central part of the Strait of Magellan near to Arthuro lake $\left(8^{\circ} \mathrm{C}\right)$, and in the Seno Almirantazgo fjord, close to glaciers Marinelli and Ainsworth $\left(8.6-9^{\circ} \mathrm{C}\right)$ ) (Table 1; Figs. 1 and 3). When comparing our data with the seasonal in-situ SST, we observe that the reconstructed SST for Southern Patagonia matches austral summer temperatures (Table 1, Fig. 3).

\section{Downcore alkenone-derived SST records}

We constructed continuous alkenone-derived SST records that cover the past $\sim 11 \mathrm{cal}$ ka BP for cores MD07-3124 (Canal Concepción; 51ํㅇ) and CHURR (Churruca fjord; $53^{\circ} \mathrm{S}$ ). We constructed SST records for the past $\sim 8 \mathrm{cal} \mathrm{ka}$ BP for core JPC-42 (Canal Wide; $50^{\circ} \mathrm{S}$ ) as alkenones were not detected in samples older than $\sim 8$ cal ka BP in that core (Figs. 1 and 4).

The mean SST value over the past $~ 8$ cal ka BP at site JPC- 42 is $12.2^{\circ} \mathrm{C}$, i.e. about $1.5^{\circ} \mathrm{C}$ higher than present-day in-situ summer temperatures (Fig. 4A; Table 1). The warmest SSTs occur during the mid-Holocene (from $\sim 6$ to $\sim 4 \mathrm{cal}$ ka BP) with values mostly $\geq 13^{\circ} \mathrm{C}$. A pronounced SST drop of $\sim 2^{\circ} \mathrm{C}$ is recorded between $\sim 0.9$ and $0.6 \mathrm{cal}$ ka BP. Over the past $600 \mathrm{yr}$, large short-term fluctuations occur with amplitudes exceeding $2^{\circ} \mathrm{C}$ and a few of such short-term events are often documented by only one data point.

For core MD07-3124 (Canal Concepción), alkenone-SSTs oscillate around a mean value of $11.5^{\circ} \mathrm{C}$ that largely resemble the present-day in-situ summer temperature (Fig. 4B; Table 1). The SST record does not reveal pronounced long-term trends, but shows a number of multi-centennial scale and shorter-term fluctuations of the order of $1-2^{\circ} \mathrm{C}$. The most important of these high frequency variations is an abrupt SST drop of $>2^{\circ} \mathrm{C}$ (from $12.7^{\circ} \mathrm{C}$ to $10.1^{\circ} \mathrm{C}$ ) at $\sim 5.4$ cal ka BP lasting until $\sim 4.9 \mathrm{cal} \mathrm{ka} \mathrm{BP}$. In the latest Holocene, since $\sim 0.9 \mathrm{cal}$ ka BP a very strong decrease in SST of ca. $2^{\circ} \mathrm{C}$ is obvious over a period of $\sim 300 \mathrm{yr}$ and SSTs remain comparatively low until the present.

Mean Holocene SST at the Churruca site is $10.2^{\circ} \mathrm{C}$ and thus close to present-day summer SST values (Fig. 4C; Table 1). Although with relative large fluctuations on a centennial timescale, SSTs are slightly colder in the early Holocene (ca. $8-11^{\circ} \mathrm{C}$, from 11.6 to $\sim 8 \mathrm{cal} \mathrm{ka} \mathrm{BP}$ ) than during the mid- to late Holocene, a time span characterized by relatively warm SSTs (range $9.5-11.6^{\circ} \mathrm{C}$ ). A cooling event occurs at $\sim 5$ cal ka BP. Similar to the Canal Wide and Canal Concepción records, a general cooling occurred after 1 cal ka BP, although it is characterized by high SST variability.

\section{Discussion}

\section{Seasonal signal of alkenone-derived SST in surface sediments}

Based on a large number of modern sediments from the global ocean, it is generally assumed that alkenone-derived SST represents mean annual SST at 0 to $30 \mathrm{~m}$ water depth (Müller et al., 1998; Conte et al., 2006). However, some studies suggest that alkenone paleotemperatures are seasonally skewed, particularly in polar latitudes (e.g., Sikes et al., 1997). Prahl et al. (2010) showed that alkenone SSTs in the subarctic NE Pacific are strongly biased towards the summer season and are $\sim 4^{\circ} \mathrm{C}$ warmer than mean annual SSTs. Prahl et al. (2010) further suggest that a summer offset of alkenone SSTs is less pronounced $\left(\sim 1^{\circ} \mathrm{C}\right)$ in the Southern Hemisphere because the seasonal temperature contrast is much less compared to the same latitude in the north. We have compared alkenone-derived SST values from surface sediments with the available in-situ SST data and the WOA 2009 dataset from the same latitude of the adjacent open ocean for austral spring and summer (Table 1; Fig. 3). In the Northern Patagonia fjords, alkenone-derived SST largely corresponds to spring in-situ temperatures, whereas in

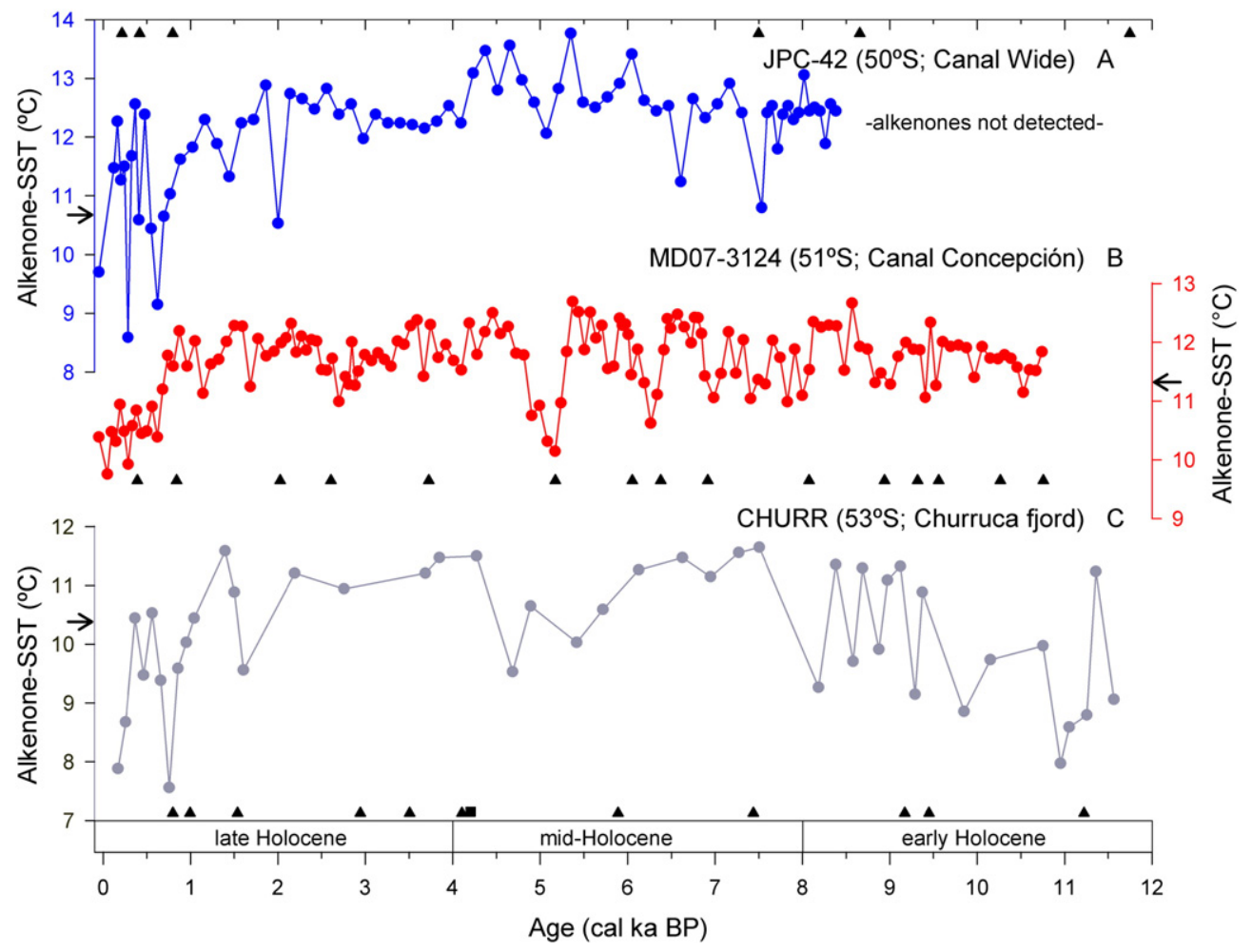

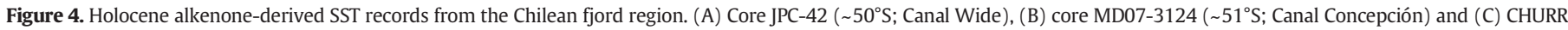

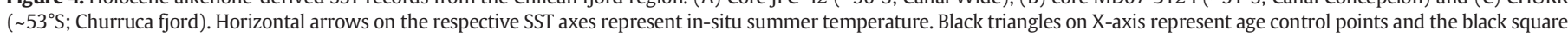
refers to the $4.15 \mathrm{cal}$ ka Mt. Burney tephra layer observed in the CHURR core (see Table 2 for details). 
Southern Patagonian fjords, alkenone SSTs are $\sim 3^{\circ} \mathrm{C}$ warmer than spring in-situ temperatures and best match austral summer temperatures. Therefore, our results for the southernmost region $\left(\sim 50^{\circ}-55^{\circ} \mathrm{S}\right)$ are consistent with the observations of Prahl et al. (2010).
Although there is only sparse information on the preferred productivity season for alkenone-producing haptophytes in the Chilean Patagonian fjords, and a strong intra-annual as well as inter-annual variability can be expected, a dominance of nanoplankton during post

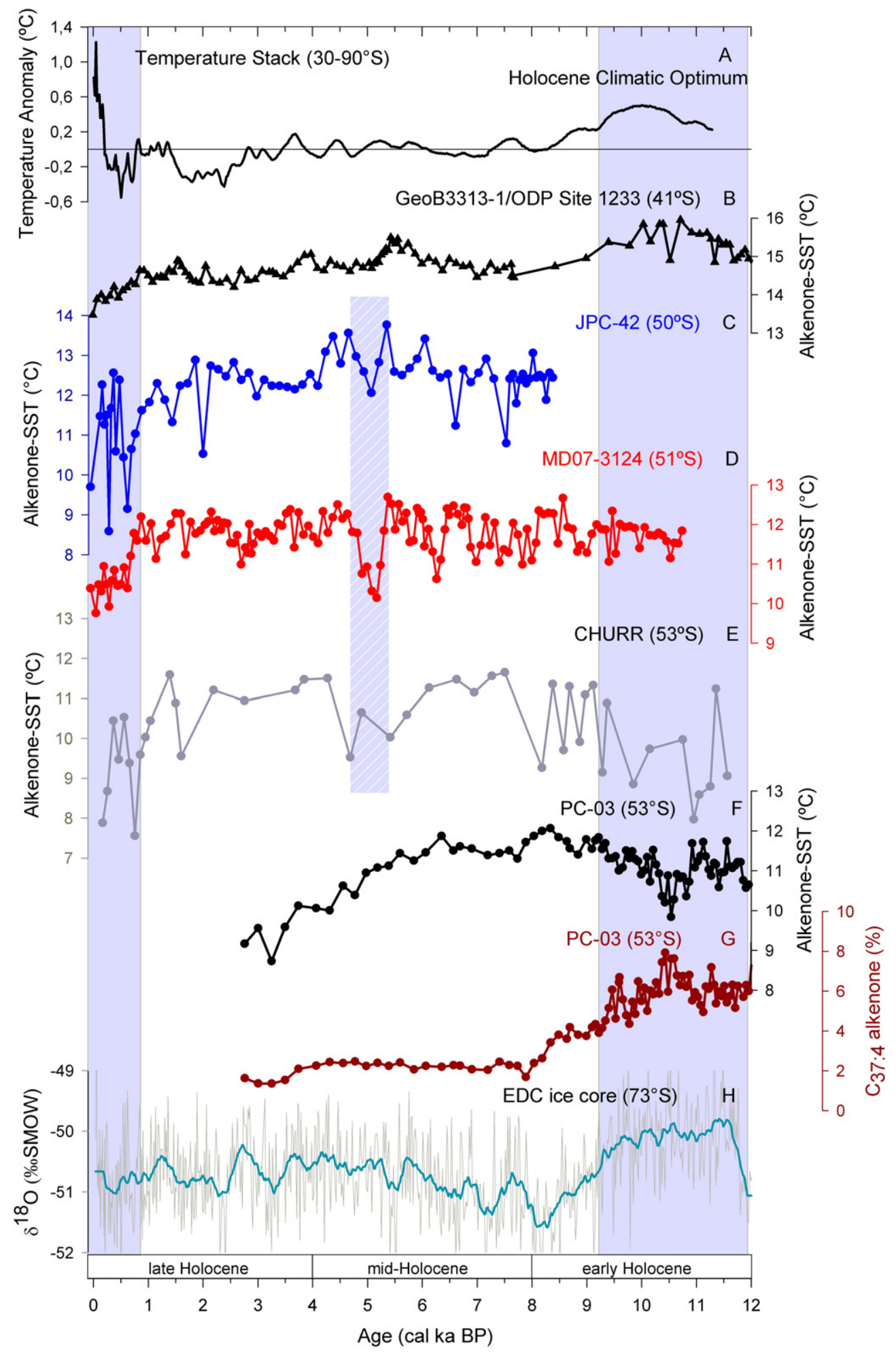

Figure 5. Holocene alkenone-derived SST off southern Chile compared to other published paleoclimatic records. (A) Anomaly of zonal mean temperature reconstruction (temperature stack) along 30-90 ${ }^{\circ} \mathrm{S}$ (Marcott et al., 2013). Alkenone-derived SST from cores (B) GeoB3313-1/ODP Site 1233 at $\sim 41^{\circ} \mathrm{S}$ (Lamy et al., 2002; Kaiser et al., 2005); (C) JPC-42 at $\sim 50^{\circ} \mathrm{S}$; (D) MD07-3124 at $~ 51^{\circ} \mathrm{S}$; (E) CHURR core at $53^{\circ} \mathrm{S}$; and (F) PC-03 at $53^{\circ} \mathrm{S}$ (Harada et al, 2013). (G) Relative abundance of tetra-unsaturated $\mathrm{C}_{37}$ alkenone (\% $\mathrm{C}_{37.4}$ alkenone) from $\mathrm{PC}-03$ core at $53^{\circ} \mathrm{S}$ (Harada et al., 2013). (H) $\delta^{18} \mathrm{O}$ record from EPICA Dome C ice core (Masson-Delmotte et al., 2011; bold line shows 300 yr moving average after interpolation to the mean resolution of ca. $20 \mathrm{yr}$ ). Gray vertical shadings denote the Holocene Climatic Optimum of the early Holocene and the cooling period of the last 0.9 cal ka BP. Gray vertical dashed shading refers to the cooling event recorded in the fjord sites at $\sim 5$ cal ka BP. See Fig. 1 for the location of the sediment cores. 
bloom events in spring in Northern Patagonia fjords, and during austral summer-autumn in Southernmost Patagonia fjords has been previously reported (Iriarte et al., 1993; Magazzù et al., 1996; Antezana, 1999; Iriarte et al., 2007; Alves-de-Souza et al., 2008; González et al., 2010, 2013). Recurrent austral summer coccolithophore blooms are found in Subantarctic Shelf waters on the Patagonian shelf/shelf break off
Argentina, with maximum growth under well-illuminated shallow mixed layer depth and low phosphate concentrations (e.g., Signorini et al., 2006; Poulton et al., 2013 and references therein). An increasing number of observations in the Southern Ocean (including e.g., satellite imagery, morphological/microscopy work) reveal coccolithophore peaks (mainly Emiliania huxleyi, an alkenone-producing species) during

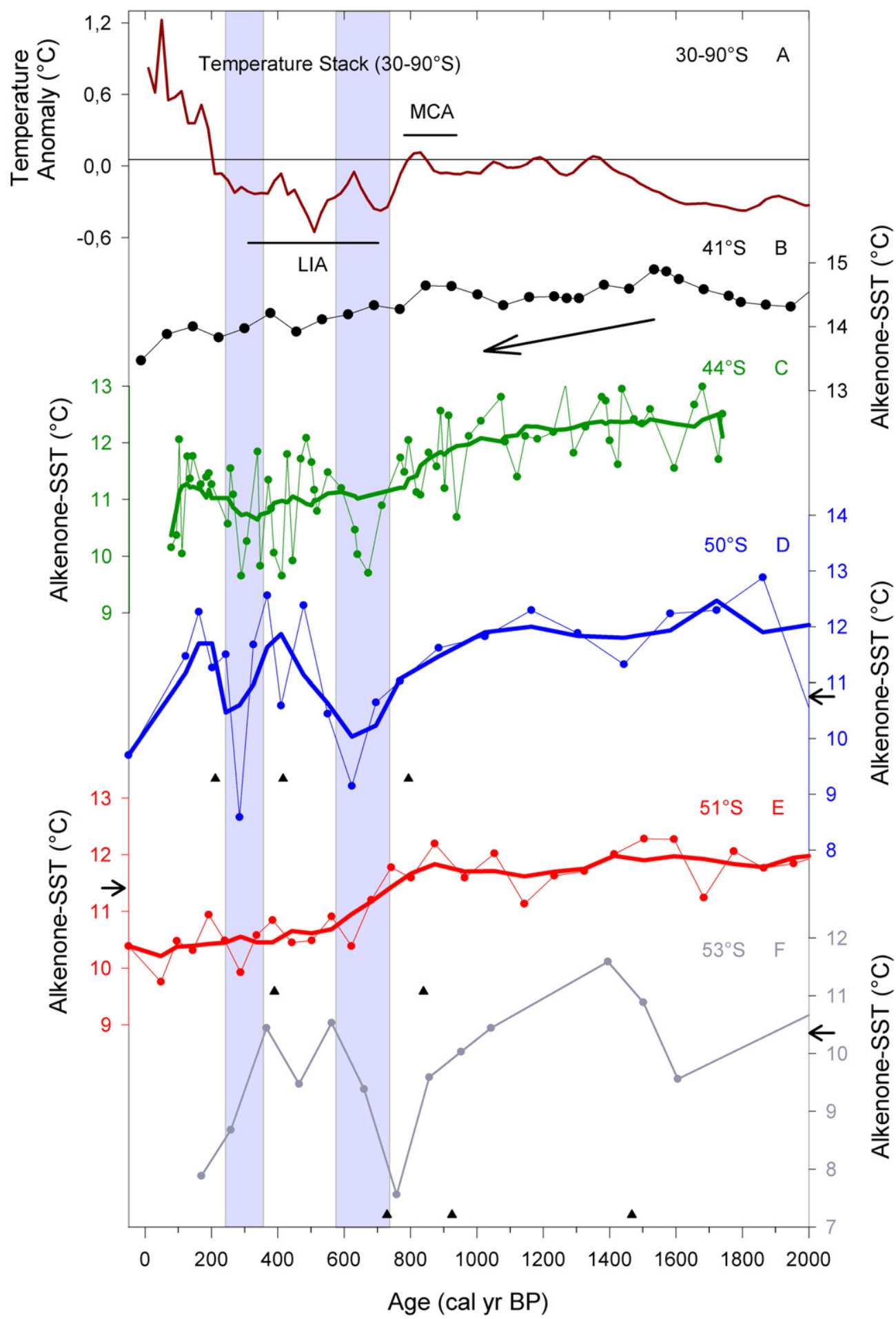

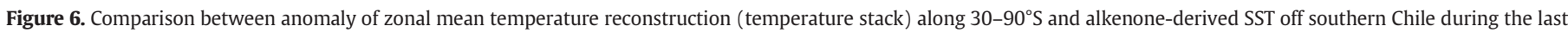

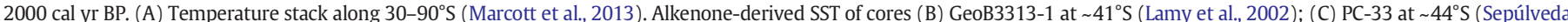

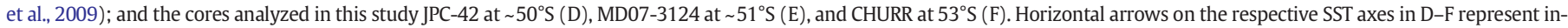

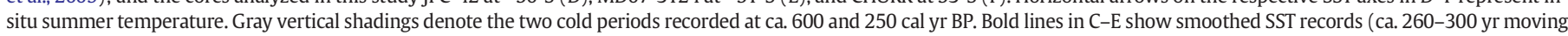
average). Black triangles on X-axis represent age control points. LIA = Little Ice Age and MCA = Medieval Climate Anomaly. 
austral summer months in surface waters of the ACC (e.g., Holligan et al., 2010; Moore et al., 2012) that are in good agreement with our results from southernmost Patagonia.

\section{Holocene SST changes}

There is widespread evidence for the presence of an early Holocene Climatic Optimum in the Southern Hemisphere. Recently, Marcott et al. (2013) showed the presence of this warm period in several terrestrial and marine records distributed between $30^{\circ}$ and $90^{\circ} \mathrm{S}$ (Fig. 5A). This warm period has also been recorded from Chilean alkenone-derived SST records at $\sim 30^{\circ} \mathrm{S}$ (Kaiser et al., 2008) and at $\sim 41^{\circ} \mathrm{S}$ (Fig. 5B) (Lamy et al., 2002; Kaiser et al., 2005), and from ice-core records in Antarctica (Fig. 5H; Masson et al., 2000; Masson-Delmotte et al., 2011). Furthermore, the early Holocene warming is likewise visible in a low-resolution alkenone SST record from the continental margin off southernmost Chile (MD07-3128; 53 S; Caniupán et al., 2011). In contrast, our new SST records from the Churruca fjord at $53^{\circ} \mathrm{S}$ (core CHURR) and Canal Concepción at 51 ${ }^{\circ} \mathrm{S}$ (core MD07-3124) display a different pattern. Core CHURR (Fig. 5E) shows relatively cold SSTs during this early Holocene warm period whereas core MD07-3124, located relatively close to the open Pacific, does not reveal a particular temperature maximum (Fig. 5D). As mentioned previously the alkenone SST record in Canal Wide at $50^{\circ} \mathrm{S}$ (core JPC-42) only extends back to 8 cal ka BP (Fig. 5C). The diverging SST pattern between the CHURR and PC-03 (Harada et al., 2013) records after about 6 cal ka BP (Fig. 5E-F), despite the fact that both cores were collected at $53^{\circ} \mathrm{S}$, is intriguing. This discrepancy could be related to the fact that the core CHURR was collected in an inner fjord under the influence of glacial meltwater input, whereas the PC-03 core was collected within the western entrance of the Strait of Magellan near Cape Pilar, being much more under the influence of open marine water (Fig. 1). The early Holocene temperature maximum in the Southern Hemisphere has been related to changes in the global ocean circulation involving a bipolar seesawlike temperature pattern (Masson et al., 2000; Lamy et al., 2010). While SST records from the open ocean may better reflect regional air temperatures, the fjords SSTs are overprinted by local processes, in particular the input of cold fresh water from snow, glacier, and drift ice melting during spring and summer, which may substantially modify the SST signal. It also has to be considered that a lower early Holocene sea level might have inhibited, to a certain extent, the inflow of comparatively warmer open ocean surface waters. Nevertheless, the presence of alkenones throughout the past 11 cal ka BP in Canal Concepción indicates marine conditions back to the early Holocene. On the other hand, the advection of colder and fresher inner fjord water (estuarine waters) was probably enhanced during the early Holocene due to higher precipitation and runoff related to stronger SWW (Lamy et al. 2010) that might additionally have kept the colder estuarine waters inside the fjord system. Indeed, the higher contribution of $\mathrm{C}_{37: 4}$ alkenone (a proxy for less saline water; e.g. Rosell-Melé et al., 2002) in the PC-03 core during the early Holocene compared to the mid- and late Holocene points to a substantial freshening of the surface waters in the area at least during the early Holocene (Fig. 5G). As well, the fact that alkenones in core JPC- 42 are only present after $\sim 8$ cal ka BP suggests considerably reduced surface water salinities in the inner Europa and Penguin fjords during the early Holocene (Figs. 1 and 5).

A pronounced cooling of $\sim 2.5^{\circ} \mathrm{C}$ lasting from $\sim 5.4$ to $4.7 \mathrm{cal} \mathrm{ka} \mathrm{BP}$ at Canal Concepción and Churruca fjord sites (Fig. 5D-E) coincides with the first, relatively limited, Neoglacial glacier advance in the Southern Andes (Glasser et al., 2004). Interestingly, the SST cooling during this event is less pronounced in our inner channel record JPC- $42\left(\sim 1.4^{\circ} \mathrm{C}\right.$; Fig. 5C) which should document even better the potential cooling effect of advancing glaciers from the surrounding Southern Patagonian Ice Field. This may suggest a Southern Ocean origin of the first Neoglacial cooling signal consistent with a sea-ice advance around Antarctica (Hodell et al., 2001; lizuka et al., 2008).

\section{The last 2000 cal yr}

The most striking features of the latest Holocene in our three new SST records are two pronounced cold events at ca. 600 and $250 \mathrm{cal} \mathrm{yr} \mathrm{BP}$ (Fig. 6D-F). Within age uncertainties, these two cold periods occurred during the global Little Ice Age (LIA), a cold period that spans much of the past millennium ( 600-300 cal yr BP) (Grove, 2001 and references therein). A regional cold interval which lasted from $\sim 700$ to about 250 cal yr BP is recorded in the temperature stack between 30 and $90^{\circ} \mathrm{S}$ (Fig. 6A; Marcott et al., 2013). This cold interval that has been interpreted, as the regional signature of the LIA, could have been associated with major glacier advances recorded in both Southern (Koch and Kilian, 2005) and Northern Patagonia (Araneda et al., 2007; Harrison et al., 2007). A similar pattern has been observed in the late Holocene alkenone-based SST record from the Jacaf Channel, located in the Northern Patagonia at $\sim 44^{\circ} \mathrm{S}$ (Fig. 6C) (Sepúlveda et al., 2009). There, the transition from warm conditions before $950 \mathrm{cal} \mathrm{yr} \mathrm{BP}$ to $\sim 2^{\circ} \mathrm{C}$ colder temperatures after $\sim 750 \mathrm{cal}$ yr BP takes place over a short period of $\sim 200 \mathrm{yr}$. Thereafter, SSTs recover rapidly to a maximum at $\sim 500 \mathrm{cal} \mathrm{yr}$ $\mathrm{BP}$ followed by rather cool but highly variable temperatures (Fig. 6C). While SSTs remain cool in the MD07-3124 record at $51^{\circ} \mathrm{S}$ over the past $600 \mathrm{yr}$ (Fig. 6E), large fluctuations occur in cores JPC-42 $\left(50^{\circ} \mathrm{S}\right)$ and CHURR $\left(53^{\circ} \mathrm{S}\right)$ (Fig. 6D, F). The SST pattern in the Concepción core is consistent with a quite continuous cooling trend as registered in the continental margin site located at $41^{\circ} \mathrm{S}$ (Fig. 6B) (Lamy et al., 2002). These results point to a stronger SST variability in the inner fjords (Fig. 6C-D, F) compared to the more oceanic location of the Canal Concepción. The SST records from the Chilean fjords and the adjacent continental margin all show a pronounced late Holocene cooling suggesting that the cooling was not a local event but a regional feature affecting an area of at least ca. $12^{\circ}$ latitude, from $\sim 41^{\circ}$ to $53^{\circ} \mathrm{S}$. The exact timing and duration of the cooling vary between sites by a few hundred years. This could possibly be a consequence of radiocarbon dating uncertainties including local reservoir age changes which are not yet well constrained in the Chilean fjords. The larger SST amplitude in the fjord records compared to those located on the continental margin suggests that these variations are possibly amplified in the fjords.

\section{Conclusions}

Surface sediment data from the complete latitudinal ranges of the Chilean fjords show that alkenone SSTs do not represent annual mean temperatures. Instead, they resemble austral spring surface temperatures in the Northern Patagonian fjords and austral summer surface temperatures in the Southern fjords, probably related to a shorter productivity season of alkenone-producing nanoplankton towards the south, in agreement with present-day productivity data.

Alkenone SSTs in our three downcore records show warmer than present temperatures over most of the Holocene except for a centennial-scale cooling centered at $\sim 5$ cal ka BP and the past $\sim 0.9$ cal ka BP. There is no evidence of a particular warming in the early Holocene as found in the Chilean continental margin records and other Southern Hemisphere temperature-related proxy data. Potential mechanisms for the absence of an early Holocene warm phase include a combination of factors such as decreased inflow of open marine waters due to shallower fjord sills towards the Pacific, enhanced advection of colder and fresher inner fjord water, and stronger westerly winds.

Two pronounced cold events of $1-2^{\circ} \mathrm{C}$ occurred in the latest Holocene at ca. 0.6 and 0.25 cal ka BP in all SST records from the Chilean fjords and offshore at the Chilean continental margin, although with a much lower amplitude. The widespread occurrence of these cold events suggests that they are not isolated events but a regional feature affecting an area of $\sim 12^{\circ}$ latitude. The higher amplitude of these changes in the fjord records points to an amplification of the signal inshore and corroborates the high sensitivity of this system for tracking regional climate changes. 


\section{Acknowledgments}

We thank the chief scientists, captains and crew members on board R/ V Marion Dufresne during IMAGES MD159/PACHIDERME cruise and RVIB Nathaniel B. Palmer during NBP0505 cruise. We are grateful to the Hydrographic and Oceanographic Service of the Chilean Navy for providing in-situ SST data. We also thank Kirsten Fahl, Walter Luttmer, Hendrik Grotheer, Ralph Kreutz and Lilian Nuñez for their assistance in the alkenone analysis, and Fabian Tapia who provided unpublished CTD data. We acknowledge the constructive comments by Dr. Heiko Moossen and one anonymous reviewer. Funding was provided by the AlfredWegener-Institut Helmholtz-Zentrum für Polar- und Meeresforschung and the Deutsche Forschungsgemeinschaft (DFG) through grants LA 1273/3-2, He3412/6-1, LA1273/5-1, and KI-456/9-1. CBL and SP acknowledge support from the COPAS Center (Project FONDAP \# 15010007 and PFB-31), and the National Oceanographic Committee (CONA) through the CIMAR-7 FIORDO Program (Grant C7F01-10 to SP).

\section{References}

Abarzúa, A., Villagrán, C., Moreno, P.I., 2004. Deglacial and postglacial climate history in east-central Isla Grande de Chiloé, southern Chile $\left(43^{\circ} \mathrm{S}\right)$. Quaternary Research 62, 49-59.

Alves-de-Souza, C., González, M.T., Iriarte, J.L., 2008. Functional groups in marine phytoplankton assemblages dominated by diatoms in fjords of southern Chile. Journal of Plankton Research 30, 1233-1243.

Antezana, T., 1999. Hydrographic features of Magellan and Fuegian inland passages and adjacent Subantarctic waters. Scientia Marina 63, 23-34.

Araneda, A.,Torrejon, F.,Aguayo, M.,Torres, L.,Cruces, F.,Cisternas, M.,Urrutia, R., 2007. Historical records of San Rafael glacier advances (North Patagonian Icefield): another clue to 'Little Ice Age' timing in southern Chile? The Holocene 17, 987-998.

Bentley, M.J.,Hodgson, D.A.,Smith, J.A.,Cofaigh, C.Ó.,Domack, E.W.,Larter, R.D.,Roberts, S.J. Brachfeld, S., Leventer, A., Hjort, C., Hillenbrand, C.-D., Evans, J., 2009. Mechanisms of Holocene palaeoenvironmental change in the Antarctic Peninsula region. The Holocene 19, 51-69.

Bianchi, C., Gersonde, R., 2004. Climate evolution at the last deglaciation: the role of the Southern Ocean. Earth and Planetary Science Letters 228, 407-424.

Breuer, S., Kilian, R., Schörner, D., Weinrebe, W., Behrmann, J., Baeza, O., 2013. Glacial and tectonic control on fjord morphology and sediment deposition in the Magellan region $\left(53^{\circ} \mathrm{S}\right)$, Chile. Marine Geology 346, 31-46.

Caniupán, M.,Lamy, F.,Lange, C.B., Kaiser, J.,Arz, H.,Kilian, R.,Baeza, O.,Aracena, C.,Hebbeln, D., Kissel, C., Laj, C., Mollenhauer, G., Tiedemann, R., 2011. Millennial-scale sea surface temperature and Patagonian Ice Sheet changes off southernmost Chile $\left(53^{\circ} \mathrm{S}\right)$ over the past 60 kyr. Paleoceanography 26, PA3221. http://dx.doi.org/10.1029/ 2010 PA002049.

Chaigneau, A., Pizarro, O., 2005. Surface circulation and fronts of the South Pacific Ocean east of 120 degrees W. Geophysical Research Letters 32, L08605. http://dx.doi.org/10. 1029/2004GL022070

Conte, M.H., Sicre, M.A., Ruhlemann, C., Weber, J.C., Schulte, S., Schulz-Bull, D., Blanz, T., 2006. Global temperature calibration of the alkenone unsaturation index (UK'37) in surface waters and comparison with surface sediments. Geochemistry, Geophysics, Geosystems 7, Q02005. http://dx.doi.org/10.1029/2005GC001054.

Divine, D.V., Koc, N., Isaksson, E., Nielsen, S., Crosta, X., Godtliebsen, F., 2010. Holocene Antarctic climate variability from ice and marine sediment cores: insights on ocean-atmosphere interaction. Quaternary Science Reviews 29, 303-312.

Garreaud, R.,Lopez, P.,Minvielle, M., Rojas, M., 2013. Large-scale control on the Patagonian Climate. Journal of Climate 26, 215-230.

Glasser, N.F., Harrison, S., Winchester, V.,Aniya, M., 2004. Late Pleistocene and Holocene palaeoclimate and glacier fluctuations in Patagonia. Global and Planetary Change 43, 79-101.

González, H.E., Calderón, M.J., Castro, L., Clement, A., Cuevas, L.A., Daneri, G., Iriarte, J.L., Lizárraga, L., Martínez, R., Menschel, E., Silva, N., Carrasco, C., Valenzuela, C., Vargas, C.A., Molinet, C., 2010. Primary production and plankton dynamics in the Reloncaví Fjord and the Interior Sea of Chiloé, Northern Patagonia, Chile. Marine Ecology Progress Series 402, 13-30.

González, H.E., Castro, L.R., Daneri, G., Iriarte, J.L., Silva, N., Tapia, F., Teca, E., Vargas, C.A., 2013. Land-ocean gradient in haline stratification and its effects on plankton dynamics and trophic carbon fluxes in Chilean Patagonian fjords $\left(47-50^{\circ} \mathrm{S}\right)$. Progress in Oceanography 119, 32-47.

Grove, J.M., 2001. The initiation of the "Little Ice Age" in the region around the North Atlantic. Climatic Change 48, 53-82.

Harada, N., Ninnemann, U., Lange, C.B., Marchant, M.E., Sato, M., Ahagon, N., Pantoja, S., 2013. Deglacial-Holocene environmental changes at the Pacific entrance of the Strait of Magellan. Palaeogeography Palaeoclimatology Palaeoecology 375, 125-135.

Harrison, S.,Winchester, V.,Glasser, N., 2007. The timing and nature of recession of outlet glaciers of Hielo Patagónico Norte, Chile, from their Neoglacial IV (Little Ice Age) maximum positions. Global and Planetary Change 59, 67-78.

Ho, S.L., Mollenhauer, G., Fietz, S., Martínez-Garcia, A., Lamy, F., Rueda, G., Schipper, K. Méheust, M., Rosell-Melé, A., Stein, R., Tiedemann, R., 2014. Appraisal of TEX ${ }_{86}$ and
$\mathrm{TEX}^{\mathrm{L}}{ }_{86}$ thermometries in subpolar and polar regions. Geochimica et Cosmochimica Acta 131, 213-226.

Hodell, D.A., Kanfoush, S.L., Shemesh, A., Crosta, X., Charles, C.D., Guilderson, T.P., 2001 Abrupt cooling of Antarctic surface waters and sea ice expansion in the South Atlantic sector of the Southern Ocean at 5000 cal yr BP. Quaternary Research 56, 191-198.

Holligan, P.M., Charalampopoulou, A., Hutson, R., 2010. Seasonal distributions of the coccolithophore, Emiliania huxleyi, and of particulate inorganic carbon in surface waters of the Scotia Sea. Journal of Marine Systems 82, 195-205.

Iizuka, Y., Hondoh, T., Fujii, Y., 2008. Antarctic sea ice extent during the Holocene reconstructed from inland ice core evidence. Journal of Geophysical Research 113 D15114. http://dx.doi.org/10.1029/2007JD009326.

Ingram, B.L.,Southon, J.R., 1996. Reservoir ages in eastern Pacific coastal and estuarine waters. Radiocarbon 38, 573-582.

Iriarte, J.L.,Uribe, J.C., Valladares, C., 1993. Biomass of size-fractionated phytoplankton during the Spring-Summer season in Southern Chile. Botanica Marina 36, 443-450.

Iriarte, J.L., González, H.E.,Liu, K.K., Rivas, C., Valenzuela, C., 2007. Spatial and temporal variability of chlorophyll and primary productivity in surface waters of southern Chile (41.5-43 $\left.{ }^{\circ} \mathrm{S}\right)$. Estuarine, Coastal and Shelf Science 74, 471-480

Jenny, B.,Valero-Garcés, B.L.,Villa-Martínez, R.,Urrutia, R.,Geyh, M.,Veit, H., 2002. Early to Mid-Holocene aridity in Central Chile and the Southern Westerlies: the Laguna Aculeo Record (34 S). Quaternary Research 58, 160-170.

Kaiser, J..Lamy, F.,Hebbeln, D., 2005. A 70-kyr sea surface temperature record off southern Chile (ODP Site 1233). Paleoceanography 20, PA4009. http://dx.doi.org/10.1029/ 2005 PA001146.

Kaiser, J.,Schefuss, E.,Lamy, F.,Mohtadi, M.,Hebbeln, D., 2008. Glacial to Holocene changes in sea surface temperature and coastal vegetation in north central Chile: high versus low latitude forcing. Quaternary Science Reviews 27, 2064-2075.

Kilian, R., Lamy, F., 2012. A review of Glacial and Holocene paleoclimate records from southernmost Patagonia $\left(49-55^{\circ} \mathrm{S}\right)$. Quaternary Science Reviews 53, 1-23.

Kilian, R., Baeza, O.,Steinke, T., Arevalo, M., Rios, C., Schneider, C., 2007. Late Pleistocene to Holocene marine transgression and thermohaline control on sediment transport in the western Magellanes fjord system of Chile $\left(53^{\circ} \mathrm{S}\right)$. Quaternary International 161 , 90-107.

Kilian, R.,Lamy, F.,Arz, H., 2013. Late Quaternary variations of the southern westerly wind belt and its influences on aquatic ecosystems and glacier extend within the southernmost Andes [Spätquartäre Variationen der südhemisphärischen Westwindzone und deren Einfluss auf aquatische Ökosysteme sowie Gletscherausdehnung in den südlichen Anden]. Zeitschrift der Deutschen Gesellschaft für Geowissenschaften $164,279-294$

Koch, J., Kilian, R., 2005. 'Little Ice Age’ glacier fluctuations, Gran Campo Nevado, southernmost Chile. The Holocene 15, 20-28.

Lamy, F.,Hebbeln, D.,Wefer, G., 1999. High-resolution marine record of climatic change in mid-latitude Chile during the last 28,000 years based on terrigenous sediment parameters. Quaternary Research 51, 83-93.

Lamy, F., Rühlemann, C., Hebbeln, D., Wefer, G., 2002. High- and low-latitude climate control on the position of the southern Peru-Chile Current during the Holocene Paleoceanography 17 (2). http://dx.doi.org/10.1029/2001PA000727.

Lamy, F., Kilian, R., Arz, H.W., Francois, J.P., Kaiser, J., Prange, M.,Steinke, T., 2010. Holocene changes in the position and intensity of the southern westerly wind belt. Nature Geoscience 3, 695-699.

Locarnini, R.A., Mishonov, A.V., Antonov, J.I., Boyer, T.P., Garcia, H.E., Baranova, O.K.,Zweng, M.M., Johnson, D.R., 2010. World Ocean Atlas 2009, Volume 1: temperature. In: Levitus, S. (Ed.), NOAA Atlas NESDIS 68. U.S. Government Printing Office, Washington, D.C. (184 pp.).

Magazzù, G., Panella, S., Decembrini, F., 1996. Seasonal variability of fractionated phytoplankton, biomass and primary production in the Straits of Magellan. Journal of Marine Systems 9, 249-267.

Maldonado, A., Villagrán, C., 2002. Paleoenvironmental changes in the semiarid coast of Chile $\left(\sim 32^{\circ} \mathrm{S}\right)$ during the last 6200 cal years inferred from a swamp-forest pollen record. Quaternary Research 58, 130-138.

Marcott, S.A.,Shakun, J.D.,Clark, P.U.,Mix, A.C., 2013. A reconstruction of regional and global temperature for the past 11,300 years. Science 339, 1198-1201.

Masson, V., Vimeux, F., Jouzel, J., Morgan, V., Delmotte, M., Ciais, P., Hammer, C., Johnsen, S. Lipenkov, V.Y., Mosley-Thompson, E., Petit, J.R.,Steig, E.J., Stievenard, M., Vaikmae, R., 2000. Holocene climate variability in Antarctica based on 11 ice-core isotopic records. Quaternary Research 54, 348-358.

Masson-Delmotte, V.,Stenni, B., Jouyel, J., 2004. Common millennial-scale variability of Antarctic and Southern Ocean temperatures during the past 5000 years reconstructed from the EPICA Dome C ice core. The Holocene 14, 145-151.

Masson-Delmotte, V., et al., 2011. A comparison of the present and last interglacial periods in six Antarctic ice cores. Climate of the Past 7, 397-423.

Mayewski, P.A., et al., 2004. Holocene climate variability. Quaternary Research 62 243-255.

McCulloch, R., Davies, S., 2001. Late-glacial and Holocene palaeoenvironmental change in the central Strait of Magellan, southern Patagonia. Palaeogeography, Palaeoclimatology, Palaeoecology 173, 143-173.

Milliken, K.T., Anderson, J.B., Wellner, J.S., Bohaty, S.M., Manley, P.L., 2009. High-resolution Holocene climate record from Maxwell Bay, South Shetland Islands, Antarctica. Geological Society of American Bulletin 26478, 1-15. http://dx.doi.org/10.1130/B26478.1.

Moore, T.S., Dowell, M.D., Franz, B.A., 2012. Detection of coccolithophore blooms in ocean color satellite imagery: a generalized approach for use with multiple sensors. Remote Sensing Environment 117, 249-263.

Müller, P.J., Kirst, G., Ruhland, G., von Storch, I., Rosell-Mele, A., 1998. Calibration of the alkenone paleotemperature index $\mathrm{U}^{\mathrm{K}^{\prime}} 37$ based on core-tops from the eastern South Atlantic and the global ocean $\left(60^{\circ} \mathrm{N}-60^{\circ} \mathrm{S}\right)$. Geochimica et Cosmochimica Acta 62 1757-1772. 
Nielsen, S.,Koc, N.,Crosta, X., 2004. Holocene climate in the Atlantic sector of the Southern Ocean: controlled by insolation or oceanic circulation? Geology 32, 317-320.

Poulton, A.,Painter, S., Young, J.,Bates, N.,Bowler, B.,Drapeau, D., Lyczschowski, E.,Balch, W. 2013. The 2008 Emiliania huxleyi bloom along the Patagonian Shelf: ecology, biogeochemistry, and cellular calcification. Global Biogeochemical Cycles 27, 1-10. http://dx. doi.org/10.1002/2013GB004641.

Prahl, F.G.,Wakeham, S.G., 1987. Calibration of unsaturation patterns in long-chain ketone compositions for paleotemperature assessment. Nature 330, 367-369.

Prahl, F.G.,Muehhausen, L.A.,Zahnle, D.L., 1988. Further evaluation of long-chain alkenones as indicators of paleoceanographic conditions. Geochimica et Cosmochimica Acta 52, 2303-2310.

Prahl, F.G., Rontani, J.-F.,Zabeti, N.,Walinsky, S.E.,Sparrow, M.A., 2010. Systematic pattern in $\mathrm{U}^{\mathrm{K}^{\prime}}{ }_{37}$ - temperature residuals for surface sediments from high latitude and other oceanographic settings. Geochimica et Cosmochimica Acta 74, 131-143.

Reimer, P.J., et al., 2013. IntCal13 and Marine13 radiocarbon age calibration curves 0-50,000 years cal BP. Radiocarbon 55, 1869-1887.

Rosell-Melé, A.,Jansen, E., Weinelt, M., 2002. Appraisal of a molecular approach to infer variations in surface ocean freshwater inputs into the North Atlantic during the last glacial. Global and Planetary Change 34, 143-152.

Schneider, C., Glaser, M., Kilian, R.,Santana, A., Butorovic, N.,Casassa, G., 2003. Weather observations across the Southern Andes at $53^{\circ}$ S. Physical Geography 24, 97-119.

Sepúlveda, J., Pantoja, S., Hughen, K.A., Bertrand, S., Figueroa, D., León, T., Drenzek, J., Lange, C., 2009. Late Holocene sea-surface temperature and precipitation variability in northern Patagonia, Chile (Jacaf Fjord, 44 S). Quaternary Research 72, 400-409.

Shevenell, A.E., Ingalls, A.E., Domack, E.W., Kelly, C., 2011. Holocene Southern Ocean surface temperature variability west of the Antarctic Peninsula. Nature 470, 250-254.

Siani, G., Michel, E., De Pol-Holz, R., DeVries, T., Lamy, F., Carel, M., Isguder, G., Dewilde, F., Lourantou, A., 2013. Carbon isotope records reveal precise timing of enhanced Southern Ocean upwelling during the last deglaciation. Nature Communications 4. http://dx.doi.org/10.1038/ncomms3758.

Sievers, H.A.,Silva, N., 2008. Water masses and circulation in austral Chilean channels and fjords. In: Silva, N., Palma, S. (Eds.), Progress in the oceanographic knowledge of Chilean interior waters, from Puerto Montt to Cape Horn. Comité Oceanográfico Nacional - Pontificia Universidad Católica de Valparaíso, Valparaíso, pp. 53-58.
Signorini, S.R., Garcia, V.M.T.,Piola, A.R.,Garcia, C.A.E., Mata, M.M.,McClain, C.R., 2006. Seasonal and interannual variability of calcite in the vicinity of the Patagonian shelf break $\left(38^{\circ} \mathrm{S}-52^{\circ} \mathrm{S}\right)$. Geophysical Research Letters 33, L16610. http://dx.doi.org/10.1029/ 2006GL026592.

Sikes, E.L., Volkman, J.K., Robertson, L.G., Pichon, J.-J., 1997. Alkenones and alkenes in surface waters and sediments of the Southern Ocean: implications for paleotemperature estimation in polar regions. Geochimica et Cosmochimica Acta 61, 1495-1505.

Silva, N Calvete C 2002 . Características oceanográficas físicas y químicas de canales australes chilenos entre el golfo de Penas y el estrecho de Magallanes (Crucero CIMAR Fiordo 2). Ciencia y Tecnología Marina 22, 23-88.

Silva, N.,Calvete, C., Sievers, H.A., 1997. Características oceanográficas físicas y químicas de canales australes chilenos entre Puerto Montt y laguna San Rafael (Crucero CIMARFiordo 1). Ciencia y Tecnología Marina 20, 23-106.

Silva, N., Calvete, C., Sievers, H.A., 1998. Masas de agua y circulación general para algunos canales australes chilenos entre Puerto Montt y laguna San Rafael (Crucero CIMARFiordo 1). Ciencia y Tecnología del Mar 21, 17-48.

Stern, C.R., 2008. Holocene tephrochronology record of large explosive eruptions in the southernmost Patagonian Andes. Bulletin of Volcanology 70, 435-454.

Strub, P.T.,Mesias, J.M.,Montecino, V.,Ruttlant, J.,Salinas, S., 1998. Coastal ocean circulation off Western South America. In: Robinson, A.R., Brink, K.H. (Eds.), The global coastal ocean: regional studies and syntheses. Wiley, New York, pp. 273-315.

Villagrán, C. 1990. Glacial climates and their effects on the history of the vegetation of Chile: a synthesis based on palynological evidence from Isla de Chiloé. Review of Palaeobotany and Palynology 65, 17-24.

Villa-Martínez, R.,Villagrán, C.,Jenny, B., 2003. The last 7500 cal yr BP of westerly rainfall in Central Chile inferred from a high-resolution pollen record from Laguna Aculeo $\left(34^{\circ} \mathrm{S}\right)$. Quaternary Research 60, 284-293.

WAIS Divide Project Members, 2013. Onset of deglacial warming in West Antarctica driven by local orbital forcing. Nature $500,440-444$.

Wanner, H., et al., 2008. Mid- to Late Holocene climate change: an overview. Quaternary Science Reviews 27, 1791-1828. 